\title{
Exitosa aplicación de un proyecto de inyección de agua en un campo de crudo pesado con pozos altamente desviados, relación de movilidad desfavorable y patronamiento irregular en Colombia
}

\author{
Successful Application of a Water Injection Project in a Heavy Crude Reservoir with \\ a Highly Deviated Well, its Relationship with Unfavorable Mobility and \\ Irregular Pattern in Colombia
}

Arango $S^{1}$; Polania $\mathbf{M}^{1^{*}}$; Giosa $\mathbf{E}^{1}$; Carrero $\mathbf{E}^{1}$; $\operatorname{Lozano}^{1}{ }^{1}$; Vega $\mathbf{R}^{1}$

Forma de citar: Arango, S.; Polanía, M.; Giosa, E.; Carrero, E.; Lozano, A. \& Vega, R. (2021). Exitosa aplicación de un proyecto de inyección de agua en un campo de crudo pesado con pozos altamente desviados, relación de movilidad desfavorable y patronamiento irregular en Colombia. Revista Fuentes, el reventón energetico, 19(1), 85-96. https://doi.org/10.18273/revfue.v19n1-2021008

\section{Resumen}

Este trabajo presenta algunas prácticas claves implementadas en un proyecto de inyección de agua, en un yacimiento de crudo pesado, con alta heterogeneidad vertical y complejidad estructural; patrones irregulares con pozos ubicados a diferentes espaciamientos y arquitectura combinada que incluye pozos horizontales, geonavegados, altamente desviados y verticales, heredados del desarrollo primario y una relación de viscosidades $(\mu \mathrm{o} / \mu \mathrm{w})$ entre 200 y 600 , lo que representa un desafío técnico y operacional.

El éxito del proyecto de inyección de agua, en estas condiciones desfavorables y complejas, ha sido el resultado de un efectivo proceso de diseño, implementación, control y monitoreo. Previo al diseño del proceso, se realizó screening de tecnología, con el fin de seleccionar el proceso de recobro más adecuado para el yacimiento sujeto de estudio; posteriormente, se realizaron pruebas de laboratorio que verificaron aspectos claves como compatibilidad de fluidos y tasas críticas de flujo; con esta información se construyó un modelo numérico 3D donde se simuló el proceso de inyección de agua y fue la base para el diseño del piloto de inyección. En el 2015 se puso en marcha un piloto compuesto de 13 patrones de inyección, que fue factor clave para probar el proceso, observar sus beneficios y corregir los comportamientos anómalos de cara a la expansión. Las lecciones aprendidas del piloto fueron: (1) la necesidad de una caracterización más detallada del yacimiento para optimizar la ubicación de los nuevos patrones y sus predicciones volumétricas; (2) un permanente manejo de la heterogeneidad vertical, mediante el uso de sartas selectivas; y (3) un muy riguroso proceso de balanceo de los patrones, mediante el seguimiento y monitoreo de las tasas de inyección a nivel de arenas, presiones de flujo y caudales de producción, para minimizar la canalización del agua y maximizar la recuperación de petróleo.

En la actualidad, se avanza en la fase de expansión de este proyecto cerrando al año 2019 con 20 patrones. Entre los años 2020-2023 se tendrá la expansión total del proyecto con alrededor de 93 patrones y en el 2021 se planea el inicio del piloto de inyección de agua mejorada (polímeros), para evaluar sus beneficios. En este proceso de expansión, el control y monitoreo de las variables como tasa y perfiles de inyección/producción, presión de inyección, calidad de agua, entre otras, han sido factores clave para anticipar desvíos en el plan y ajustarlos a condiciones más favorables que permitan tener una mejor eficiencia del proceso.

El incremento actual en el factor de recobro de algunos patrones es del orden de $2 \%$ y la expectativa es llevarlo cerca del $6 \%$ mediante la inyección de un volumen poroso en 20 años. Ya se han incorporado cerca de $90 \mathrm{MM}$ de barriles de reservas $3 \mathrm{P}$ asociadas a este proyecto. Los resultados de desempeño han sido evaluados y comparados con modelamiento analítico y numérico, evidenciando un comportamiento mejor al esperado.

Palabras clave: Inyección de Agua; Crudo Pesado; Factor de Recobro; Sarta Selectiva; Patrones Irregulares.

\footnotetext{
${ }^{1}$ Ecopetrol, Carrera 13 No. 36 24, Bogotá, Colombia.

* maria.polania@ecopetrol.com.co
} 


\begin{abstract}
This work presents some key practices implemented in a water injection project on a heavy crude reservoir with high vertical heterogeneity and complex structure. Irregular patterns with wells located at different spaces, and a combined architecture that includes horizontal, geo-navigated, highly deviated, and vertical wells, are inherited from primary development and a viscosity ratio $(\mu o / \mu w)$ between 200 and 600, representing a technical and operational challenge.
\end{abstract}

The success of the water injection project, under these unfavorable and complex conditions, has been the result of an effective design, implementation, control, and monitoring process. Before carrying out the design of the process, a technological screening was performed to select the most appropriate recovery process for the understudy reservoir. After that, some laboratory tests were carried out verifying fluid compatibility and critical flow rates. With this information, a 3D numerical model was built where the water injection process was simulated and become the basis for the injection pilot design. In 2015, a pilot consisting of 13 injection patterns was launched, which was the key factor in testing the process, observing its benefits, and correcting the anomalous behaviors in the expansion face. The lessons learned from this pilot were: (1) The need for a more detailed characterization of the reservoir to optimize the location of the new patterns and their volumetric predictions. Also, (2) the permanent management of vertical heterogeneity using selective strings. Finally, (3) a very rigorous process of balancing the patterns by monitoring the injection rates at the sand level, flow pressures, and production flows to minimize the water channeling and maximize oil recovery.

Nowadays, in the expansion phase, there has been a lot of progress closing 2019 with 20 employers in this project. Between 2020 and 2023, this project will be fully expanded with about 93 patterns, and in 2021 is planned to start the pilot of improved water injection (polymers) to evaluate its benefits. In this expansion process, the variables control and monitoring such as, injection/production rate and profiles, injection pressure, water quality, among others have been crucial factors to anticipate plan deviations and adjust them to better conditions that allow greater process efficiency.

The current increase in the recovery factor of some employers is $2 \%$, and the expectation is to bring this percentage close to $6 \%$ by injecting a porous volume in 20 years. About $90 M M$ barrels of $3 P$ reserves associated with this project have already been incorporated. The performance results have been evaluated and compared with analytical and numerical modeling showing a better behavior than expected.

Keywords: Waterflood; Heavy Oil; Recovery Factor; Selective String; Irregular Patterns.

\section{Introducción}

Castilla es un campo localizado en la cuenca de los Llanos Orientales en Colombia, que produce crudo pesado de $12^{\circ}$ API proveniente de dos formaciones denominadas $\mathrm{K} 1$ y $\mathrm{K} 2$, a profundidades de entre 7000 y 8000 pies TVD. La formación más profunda, $\mathrm{K} 2$, asociada a depósitos de tipo fluvial a trenzados deltaicos, produce bajo el soporte de un acuífero activo muy potente; mientras que $\mathrm{K} 1$ corresponde a una secuencia deltaica dominada por mareas, y produce principalmente por descompresión de roca y fluidos, con un menor aporte del acuífero. Con el objeto de detener la declinación de presión que se empezó a evidenciar en la formación K1 Inferior (subunidad de la formación K1) e incrementar el bajo factor de recobro que se anticipa, se implementó un piloto de inyección de agua en julio de 2015, consistente en dos patrones irregulares, cada uno con un inyector, y entre 8-9 productores asociados en su primera línea. La información preliminar suponía que, dada la excelente calidad de roca, el perfil de inyección sería bastante homogéneo. Sin embargo, la información de registros de producción e inyección evidenció una distribución preferencial de la inyección en ciertos ciclos estratigráficos, lo cual mostró la necesidad de completamientos selectivos con el fin de mejorar la eficiencia vertical de barrido. (Mantilla, J., Rojas, D. 2019)

Debido a la irregularidad de los patrones, se presentó una rápida canalización en las arenas de mejor calidad de los pozos cercanos a los inyectores. Esta anomalía se logró mitigar reduciendo de manera significativa las tasas de inyección en estas arenas, permitiendo una mejor distribución vertical del barrido, demorando la irrupción de agua en los pozos y permitiendo obtener incrementales de producción de petróleo en los pozos beneficiados por la inyección.

\section{Definición de la alternativa}

El estudio de selección de alternativas para el campo Castilla se realizó mediante la herramienta computacional ecoEOR, la cual permite identificar el método EOR técnicamente aplicable al yacimiento en estudio, de acuerdo con los criterios de diferentes autores. De manera complementaria, se emplearon yacimientos análogos a nivel global donde este tipo 
de tecnología ha sido implementada y los resultados han sido favorables. La comparación con análogos existentes proporciona un sustento para la aplicación de pilotos, a pesar de evidenciar la no viabilidad a nivel teórico. Con base en las propiedades de la unidad K1 Inferior, en la tabla 1 se resumen los resultados para 9 tecnologías de recobro mejorado no térmico.

Tabla 1. Resultados screening campo Castilla.

\begin{tabular}{|c|c|}
\hline $\begin{array}{c}\text { Campo - Fm: CASTILLA } \\
\text { Guadalupe Superior (k1 Inf) }\end{array}$ & Resultado \\
\hline Inyección de Agua & Análogo exitoso \\
\hline Inyección de Polímero & Cumple \\
\hline Inyección Surfactante Polímero & No cumple \\
\hline Inyección $\mathrm{CO}_{2}$ Miscible & No cumple \\
\hline Inyección $\mathrm{CO}_{2}$ Insmiscible & Cumple \\
\hline Inyección Gas Pobre & No cumple \\
\hline Inyección Gas Rico & No cumple \\
\hline Inyección de Nitrógeno & No cumple \\
\hline WAG & Análogo exitoso \\
\hline
\end{tabular}

En la tabla 2 se comparan las propiedades del campo en estudio con algunos criterios reportados en la literatura, considerados óptimos para la aplicación de un proceso de inyección de agua; como se observa, cuatro de los criterios no se satisfacen. Es claro que la inyección convencional de agua está recomendada y se ha implementado ampliamente en yacimientos donde la razón de movilidad agua/aceite es favorable $(<5)$, es decir, donde no se generen efectos importantes de digitación viscosa, y se produzca un desplazamiento eficiente del aceite. No obstante, existen campos parcialmente análogos, donde la tecnología se ha implementado con resultados satisfactorios, ejemplo de ellos son los campos Court, Pelican Lake, Mooney, Brintnell, Suffield, y Wildmere (Canadá) y Chichimene (Colombia), dichas evidencias prácticas dieron soporte a la ejecución de la inyección de agua en el campo Castilla. (Delamaide, E. 2018) (Beliveau, D. 2008) (Delamaide, E. 2014) (Beliveau, D. 2009) (Jackson, R. 1968)

Tabla 2. Datos y criterios empleados para screening de inyección de agua - Castilla K1_Inf.

\begin{tabular}{lllr}
\hline & Valor & \multicolumn{1}{c}{ Autor } & Campo de estudio:Castilla \\
\hline Gravedad API del crudo & $>15$ & E.C Donalson - 1985 & 12.5 \\
Saturación de aceite al inicio del proyecto & $>40$ & Ganesh C. Thakur - 1998 & 52.5 \\
Saturación actual de agua & $<50$ & Ganesh C. Thakur - 1998 & 0 \\
Saturación de gas al inicio del proyecto & $<30$ & Petroleum Engin. Handbook - 1987 & 71 \\
Saturación de agua irreducible & $<50$ & Ganesh C. Thakur - 1998 & $500-2000$ \\
Espesor neto & NC & Ganesh C. Thakur - 1998 & 185 \\
Permeabilidad & NC & Ganesh C. Thakur - 1998 & $20-25$ \\
Temperatura de yacimiento & NC & Ganesh C. Thakur - 1998 & 49.95 \\
Porosidad & NC & Ganesh C. Thakur - 1998 & Arenas Arcillosas \\
Razón de movilidad agua aceite & $0.2-5$ & William Cobb - 2001 & Si \\
Litología & Arsenica o Caliza & Petroleum Engin. Handbook - 1987 & No \\
Acuifero? & No & ECP - SYA - WF - 2011 & ECP - SYA - WF - 2011 \\
Capa de Gas? & No & &
\end{tabular}

La tabla 3 presenta un comparativo entre las propiedades del campo Castilla con las del campo Court en Canadá. A nivel general, se evidencia que Castilla es un yacimiento más profundo, con un aceite más pesado y una temperatura de yacimiento que duplica la del otro campo; al mismo tiempo, se observa que en el momento de iniciar el proceso de inyección de agua, el factor de recobro alcanzado en el campo Court es menor al del campo en estudio. (Kantzas, M., Kantazs A. 2007) (Kantzas, M., Kantzas A. 2008) 
Exitosa aplicación de un proyecto de inyección de agua en un campo de crudo pesado con pozos altamente desviados, relación de movilidad desfavorable y patronamiento irregular en Colombia

Tabla 3. Propiedades Principales del Campo Court y el Campo Castilla.

\begin{tabular}{lcc}
\hline \multicolumn{1}{c}{ Parámetro } & Campo Court & Campo Castilla \\
\hline Ubicación & West Central Saskatchewam, Canadá & Llanos Orientales, Colombia \\
Profundidad, ft & 2854 & 5249 \\
Gravedad API & 17 & 15,5 \\
Viscosidad de crudo, cp & 155 & 150 \\
Saturación de aceite, $\%$ & 50 & 52,5 \\
Permeabilidad, mD & 2100 & $500-2000$ \\
Porosidad, $\%$ & 29 & $20-25$ \\
Temperatura de yacimiento, ${ }^{\circ} \mathrm{F}$ & 86 & 185 \\
Presión de yacimiento, psi & 646 & 2400 \\
Inicio de Inyección & Mayo, 1988 & Julio, 2015 \\
Factor de recobro inicio inyección, $\%$ & 2 & 4 \\
Factor de recobro alcanzado, $\%$ & 24 & 4
\end{tabular}

\section{Descripción geológica}

Geológicamente, el campo Castilla se encuentra localizado en el sector suroccidental de la cuenca de los Llanos Orientales, subcuenca Apiay-Ariari, en el sector del "foreland", y está asociado a una zona de moderada complejidad estructural. El campo corresponde a un cierre estructural en tres direcciones contra el sistema de fallas de Castilla. La estructura está representada por un gran anticlinal de aproximadamente $12 \mathrm{~km}$ de largo por $5 \mathrm{~km}$ de ancho y dirección de cabeceo hacia el nororiente.

El modelo estructural del campo Castilla más reciente fue realizado con base en la interpretación del cubo sísmico de $118 \mathrm{~km}^{2}$, adquirido en el año 2002, reprocesado en el 2010 y ajustado con la información de topes estructurales de 710 pozos perforados a septiembre del 2018 (véase figura 1).

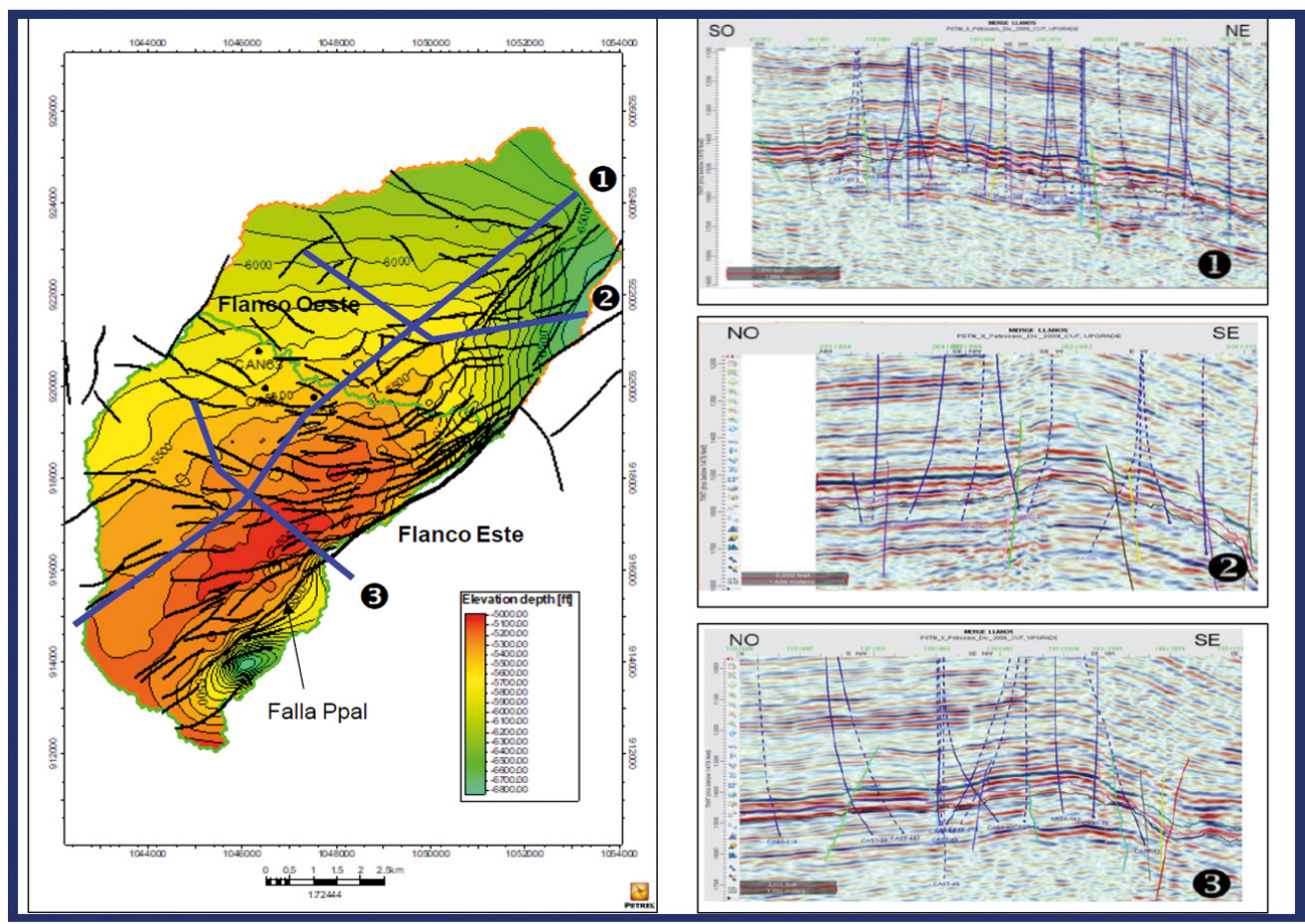

Figura 1. Mapa estructural al tope de la formación K1 Inferior. 
La estructura se encuentra afectada por una serie de fallas normales con dirección nororiente/suroccidente, algunas asociadas a la falla principal. Casi la totalidad de las fallas interpretadas son normales y el régimen tectónico es estrictamente extensional, con alguna influencia transpresiva.
Los principales yacimientos productores pertenecen a la formación Guadalupe de edad cretácica, correspondientes a las unidades K1 Inferior y K2. La formación San Fernando (unidad T2), de edad terciaria, y las unidad K1 superior/K1 medio también son consideradas objetivos potenciales de desarrollo (véase figura 2).

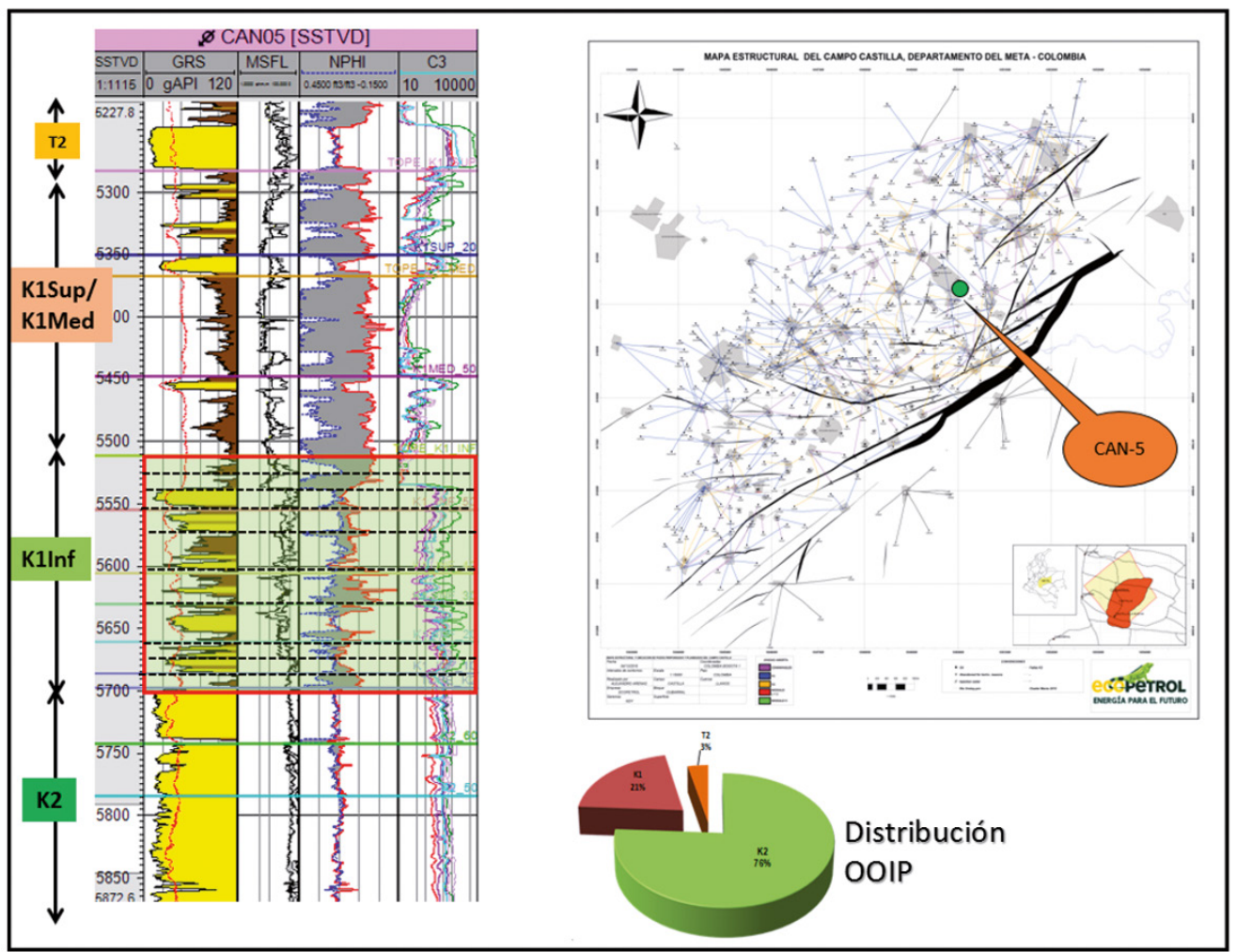

Figura 2. Columna estratigráfica horizontes productores campo Castilla y distribución del OOIP. Fuente: Ecopetrol, 2018.

Desde el punto de vista estratigráfico, la unidad K1 Inferior está enmarcada en un proceso marino transgresivo a través de una planicie costera extensa, dando lugar a la acumulación de arenas y arcillas en ambientes estuarinos y de plataforma somera, tales como canales fluviales con influencia mareal, canales distributarios, crevasse, frentes de delta y sedimentos propios de bahías estuarinas, barras de marea y plataforma lodosa. Al igual que el reservorio $\mathrm{K} 2$, todas las arenas de la unidad K1 Inferior presentan excepcional calidad de roca, en especial los depósitos asociados a la plataforma somera y frentes deltaicos. La unidad K1 Inferior se encuentra subdividida en 17 ciclos sedimentarios. La figura 3 ilustra el modelo de depositación de la unidad K1 Inferior. (Ecopetrol, 2018)

\section{Comportamiento de presión}

En principio, el foco del desarrollo de Castilla fue la formación $\mathrm{K} 2$, debido a su productividad y tamaño. Cuando el desarrollo tuvo mayor enfoque en K1, se empezó a observar una declinación en la presión de yacimiento que evidenció la pobre conexión de este yacimiento con el acuífero activo y la necesidad de implementar algún tipo de mecanismo de soporte de presión de yacimiento, con el fin de mejorar su oportunidad de recobro (véase figura 4) y compensar la extracción a la que se estaba exigiendo esta formación. La implementación del piloto de inyección de agua inicia por el área central del campo, donde la caída de presión se mostraba más crítica (véase figura 5). (Ecopetrol, 2018) 

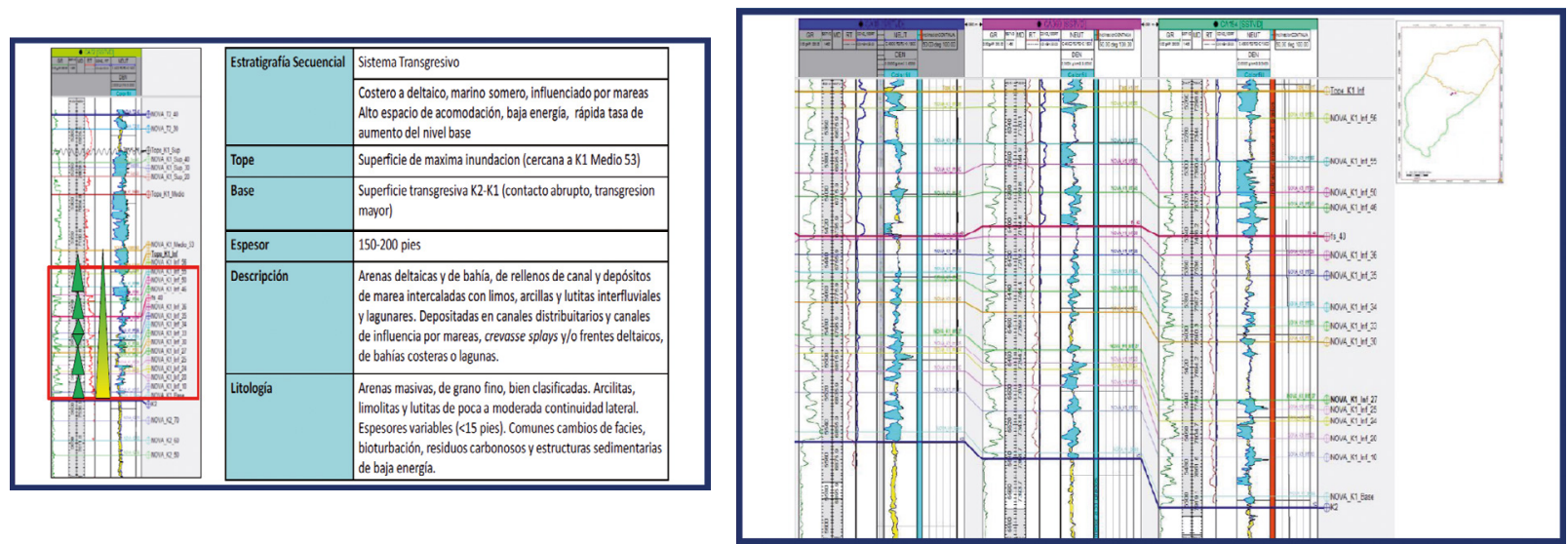

Figura 3. Modelo de depositación Unidad K1 Inferior. Fuente: Ecopetrol, 2018.

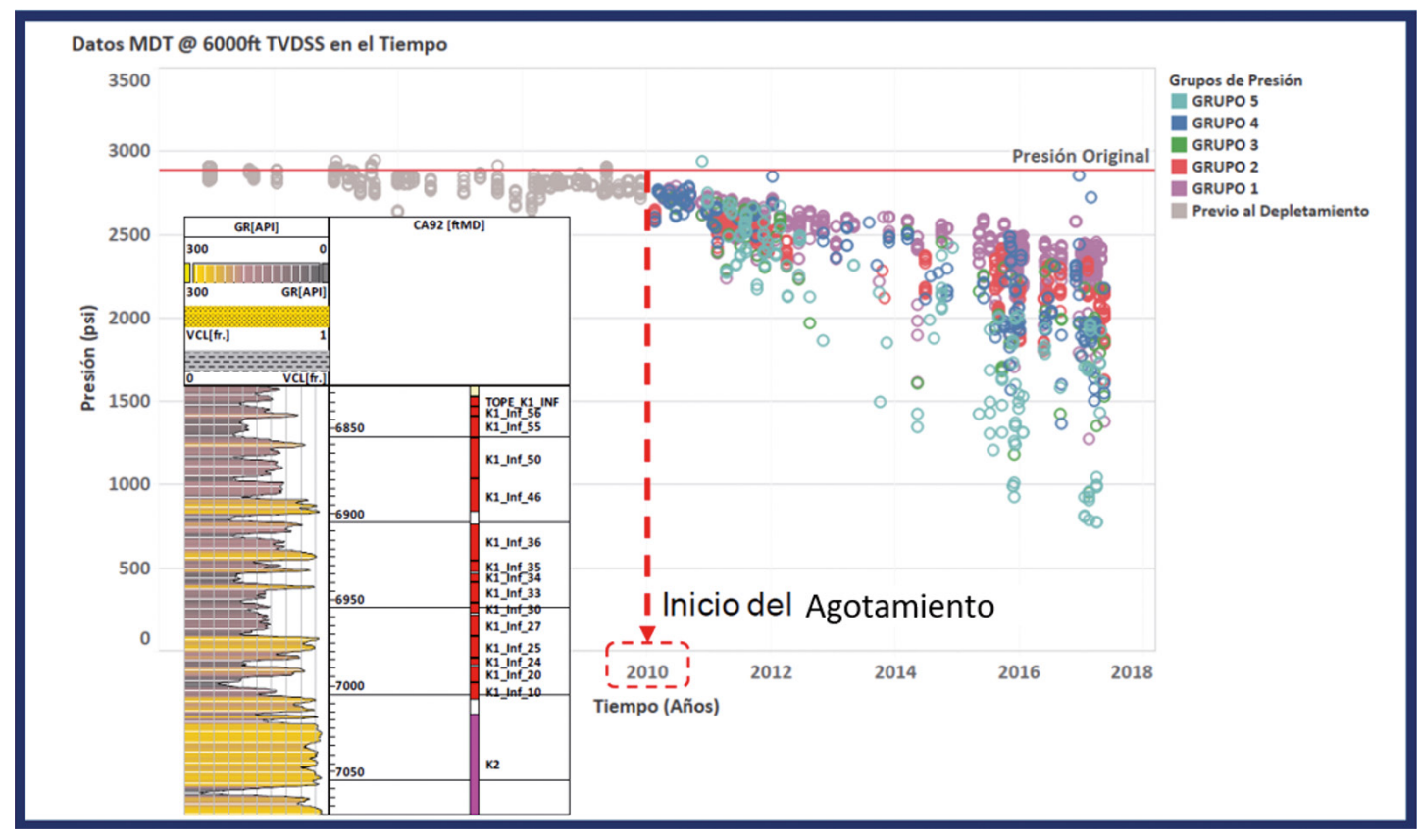

Figura 4. Comportamiento de presión en la formación K1 Inferior.

\section{Pruebas de laboratorio}

Estudios de laboratorio se efectuaron con el fin de determinar la factibilidad de la inyección de agua en la formación K1_Inferior, los cuales incluyeron sensibilidad a las aguas de inyección y tasa crítica de flujo, y mostraron valores aceptables respecto a la compatibilidad de la formación a la inyección y ningún problema respecto a tasas crítica de inyección o de producción.

El estudio realizado en el 2012 mostró que la tendencia del agua de formación a generar escamas disminuye al mezclarse con el agua de la estación Castilla 2, que será utilizada como agua de inyección; por lo tanto, se concluye que el agua a utilizar es compatible con el agua de formación. Por otro lado, las pruebas de compatibilidad entre el agua de inyección de la estación Castilla 2 y la formación mostraron que al someter diferentes muestras de roca del yacimiento al fluido de inyección, alcanzando tasas de hasta $32 \mathrm{~cm}^{3} /$ min, no se evidencia reducción en la permeabilidad asociada a daño de formación. (ICP, 2009) (Ecopetrol 2012) (ARC Group, 2010) (ARC Group, 2015) 


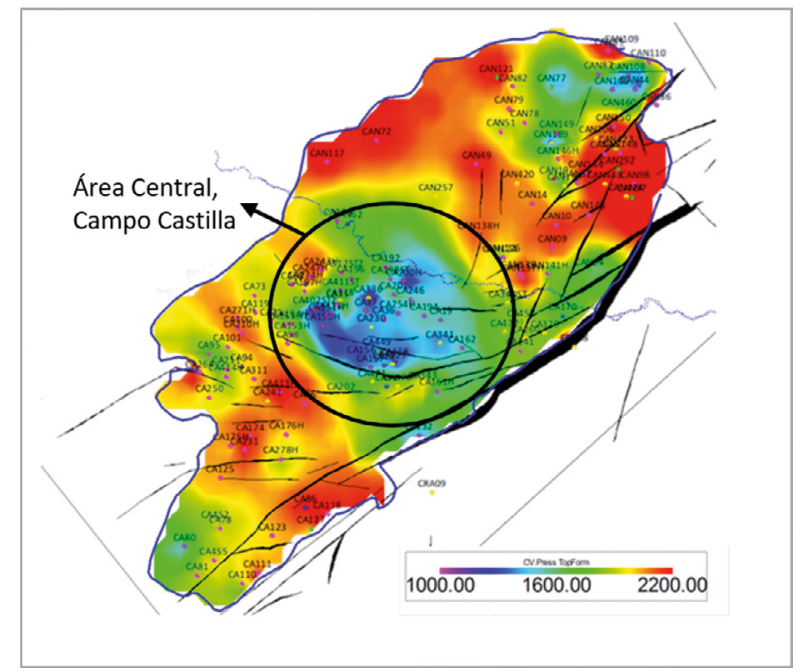

Figura 5. Distribución areal de presión estática en la formación K1 Inferior previo al proyecto de inyección de agua.

\section{Simulación numérica}

Con el fin de evaluar el volumen potencial incremental del proceso de inyección de agua en la formación $\mathrm{K} 1$, se realizaron corridas predictivas de simulación numérica, las cuales tuvieron en cuenta las siguientes consideraciones.

- Caso base: mantenimiento de las condiciones operativas de los pozos existentes a mediados de 2013, es decir, un escenario en el que se no se implemente un proyecto de inyección de agua, ni se incremente el número de pozos del campo.

- Caso perforación primaria K1 y K2: desarrollo primario (perforación) en las unidades K1 y K2.

- Caso inyección de agua en K1: inyección de agua con un patronamiento inicial de 56 inyectores (véase figura 6).

Adicionalmente, los análisis de ingeniería básica y el ajuste histórico del modelo de simulación indican que debido a la pobre interacción del acuífero con la formación productora K1 Inferior, la presión de yacimiento podría caer hasta 1200 psi, lo que afectaría la productividad y el recobro final por pozo; razones suficientes para comenzar a evaluar la implementación de un proyecto de recobro secundario que fuera eficiente tanto en la recuperación de petróleo por efecto de barrido areal y vertical, como en el mantenimiento de presión en la unidad productora

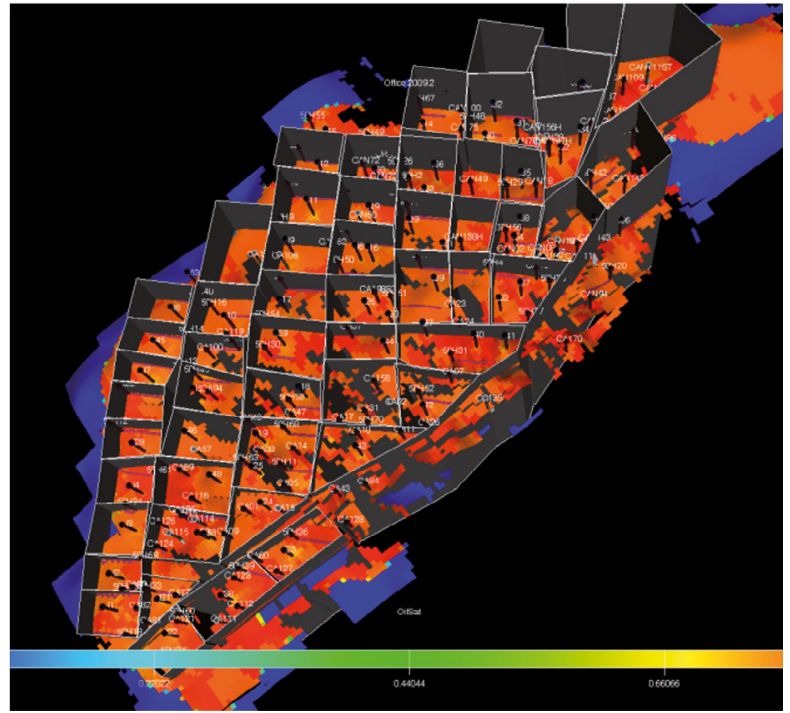

Figura 6. Visión inicial del patronamiento.

Los resultados del modelo numérico se muestran en la figura 7 , en donde se observa un incremental de petróleo por efecto de la inyección de agua en K1 de aproximadamente $5 \%$ con respecto al caso de la campaña de perforación (desarrollo primario).

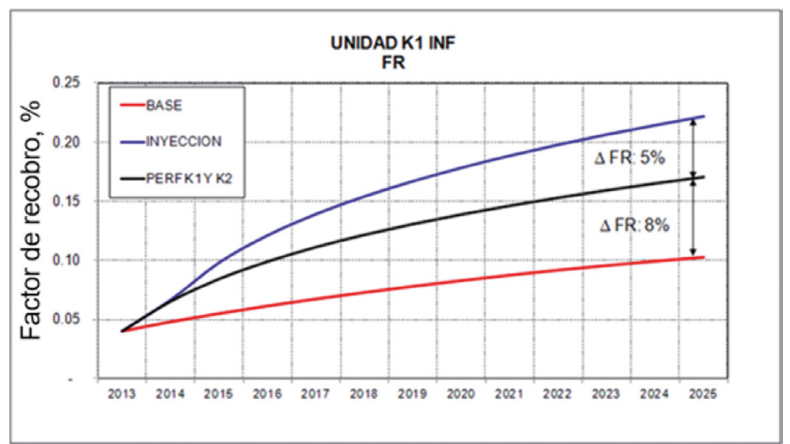

Figura 7. Factor de recobro - caso con inyección de agua.

A partir de estos análisis, se seleccionaron 13 patrones de inyección para ser ejecutados como pilotos para corroborar en campo los resultados preliminares de simulación (véase figura 8 ).

Los criterios de selección que se tuvieron en cuenta fueron los siguientes:

- Cercanía a línea de vertimiento y línea estaciones Castilla 2 y Acacias, logrando una optimización de la infraestructura existente por las limitaciones de las condiciones del entorno.

- Áreas representativas con propiedades petrofísicas promedio. 
- Presión actual del yacimiento.

- Zona con pozos abiertos en K1 Inferior, independiente o commingled.

- Aprovechamiento de pozos cerrados o de baja producción.

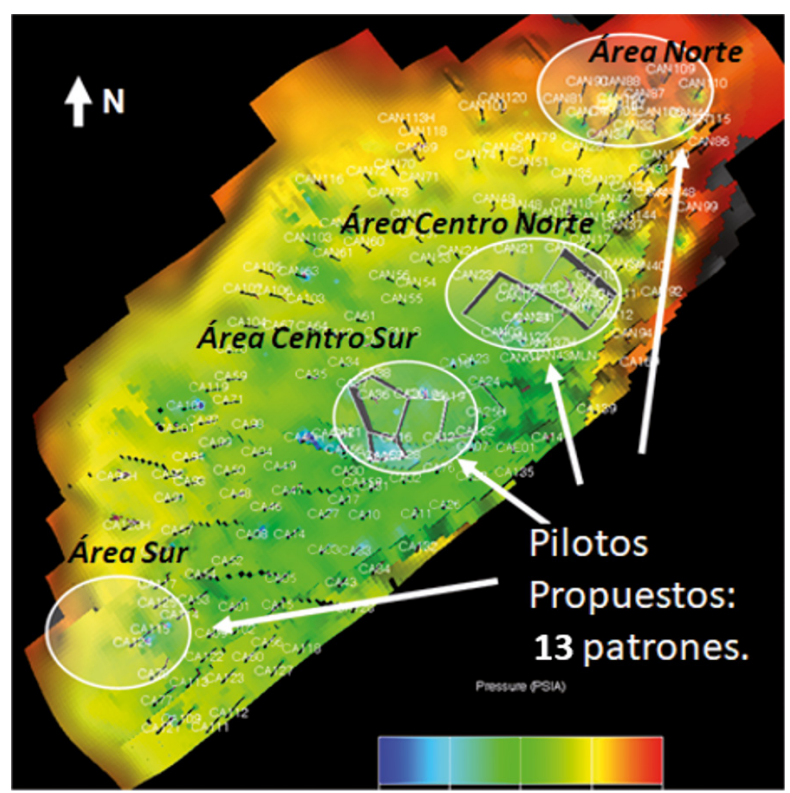

Figura 8. Ubicación de los pilotos de inyección de agua.

Entre las incertidumbres asociadas al proyecto de inyección y que fueron evaluadas mediante la implementación de los pilotos se encontraban:

- Inyectividad por pozo: caudal y presión en cabeza.

- Irrupción temprana de agua: diferencia de movilidad.

- Tiempo de respuesta a la inyección.

- Respuesta a la inyección, en la unidad K1 en pozos productores commingled (pozos con más de una formación abierta). Donde se encuentra abierta la unidad K2 se tiene un diferencial de potencial.

- Estado actual de los pozos a convertir y productores, especialmente en términos de integridad mecánica (cemento).

- Necesidad de trabajos de estimulación tanto en productores como en inyectores.
Los patrones de inyección son de geometría irregular y están conformados por productores horizontales geonavegados selectivamente a los ciclos estratigráficos de K1 Inferior o productores altamente desviados e inyectores verticales. Adicionalmente, se realizaron sensibilidades de tasas de inyección de agua, considerando velocidades de flujo que permitieran desplazar un volumen poroso en cada patrón entre 10 y 20 años, dando como resultado un rango de factor de recobro incremental entre $3 \%$ y $6,4 \%$, tal como se muestra en la figura 9.

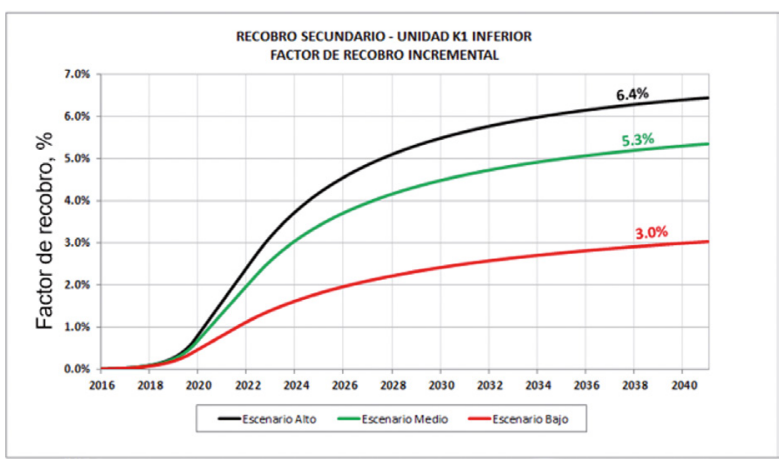

Figura 9. Factor de recobro inyección de agua.

Teniendo en cuenta estos resultados del modelamiento numérico, se realizó una conceptualización inicial del proyecto, el cual estaría conformado por 56 patrones de inyección de agua. Las condiciones de inyección propuestas para los inyectores entre los 7000-8500 bwipd (barriles de agua inyectados por día), con un límite máximo de presión en fondo de 5000 psi, teniendo en cuenta una presión de inyección en cabeza de máximo 2000 a 2500 psi y con el objetivo de no sobrepasar el gradiente de fractura estimado en 0,7 $\mathrm{psi} / \mathrm{ft}$, según los trabajos de fracturamiento realizados en el campo. Sin embargo, uno de los parámetros a validar con la implementación del piloto sería la tasa de inyección por pozo, por los riesgos de canalización.

\section{Análisis de desempeño del piloto de inyección}

La implementación del piloto se estructuró bajo los parámetros del Procedimiento para la Planeación, Ejecución y Cierre de Pilotos de Recobro (procedimiento estándar de Ecopetrol, abril de 2018 última versión), que incluye las fases descritas previamente: caracterización, selección, definición, ejecución, operación y cierre. 
Los resultados de la implementación del piloto que se han observado hasta el momento son favorables: en términos de producción incremental, a julio de 2019, el acumulado de petróleo es de 4,1 MMbls (el agua inyectada es de 16,8 MMbls), y en sostenimiento de presión, lo cual se evidencia en una reducción de la tendencia de declinación y en el ajuste de las tasas de inyección.

La figura 10 presenta el comportamiento de producción de los pozos beneficiados por la inyección durante la fase piloto del proyecto. La línea negra muestra el comportamiento de la tasa de líquido y la azul punteada el corte de agua. Se observa en color verde oscuro el volumen que se habría obtenido sin inyección; en marrón, la producción incremental obtenida por secundaria; y en verde claro, la producción incremental proyectada. En la figura 11 se observa el comportamiento de las tasas de inyección (línea azul) y el promedio de las presiones en cabeza de los pozos inyectores (línea roja).

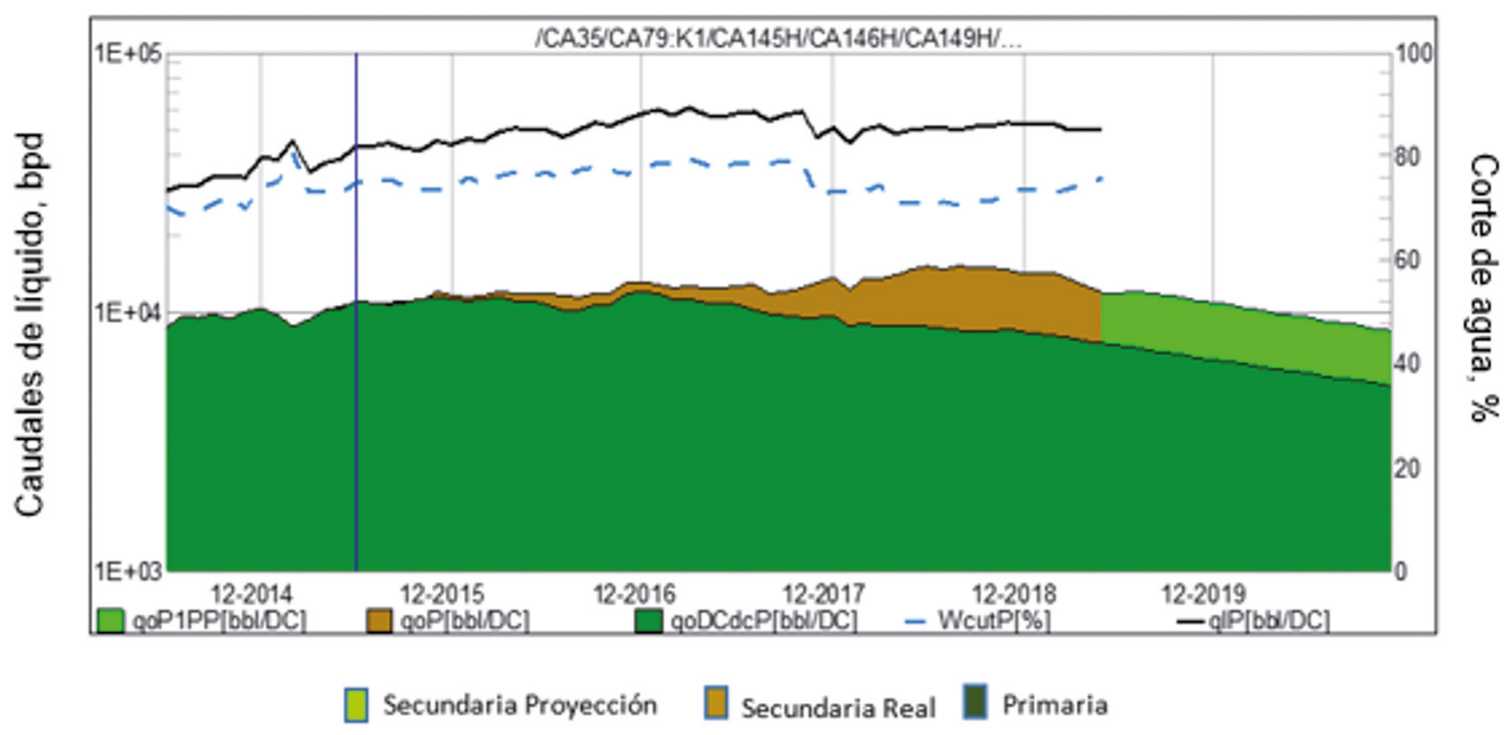

Figura 10. Comportamiento de producción patrones existentes.

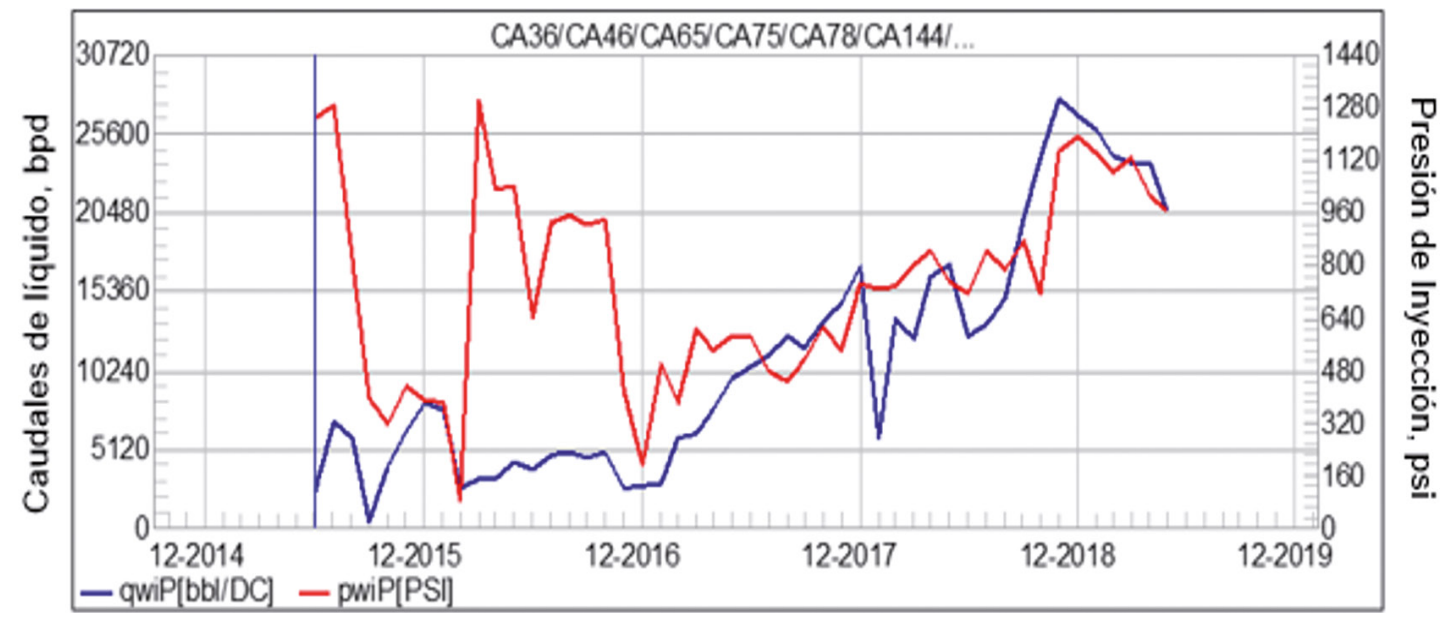

Figura 11. Caudal y presión promedio de inyección patrones existentes. 
La figura 12 muestra la ubicación de los 13 patrones del piloto de inyección, más los 6 patrones de expansión temprana. En blanco, el esquema de patrones para la futura expansión del proyecto.

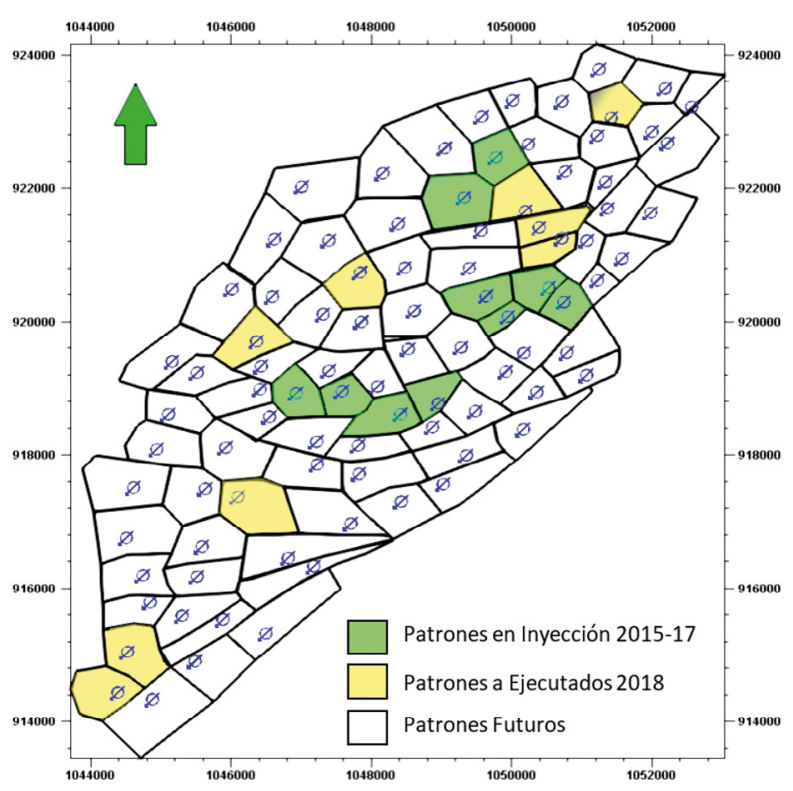

Figura 12. Ubicación patrones existentes y futuros.

\section{Monitoreo y control}

Uno de los factores clave durante el proceso ha sido el seguimiento de diferentes variables de control, tanto en pozos productores como en inyectores. A través de reuniones de trabajo que se llevan a cabo periódicamente con todos los equipos involucrados, se realiza un seguimiento detallado de los reportes obtenidos por parte de las compañías aliadas, acerca de la calidad de agua tomada a la salida de las estaciones y en cabeza de pozo, lo que permite tomar acciones preventivas y remediales en el momento de observar alguna desviación de los parámetros evaluados respecto a los valores tomados como guía.

Así mismo, se realiza una revisión de los perfiles de inyección, para verificar la eficiencia vertical del proceso, y se analiza el comportamiento de los productores con el propósito de identificar oportunidades de cambios en las variables de extracción que permitan mejorar la eficiencia areal de cada patrón de inyección.

\section{Lecciones aprendidas}

Durante el desarrollo del piloto de inyección del campo Castilla se evidenciaron las siguientes condiciones, cuyo entendimiento permitió mejorar la eficiencia del proceso de inundación para la expansión:

- Complejidad geológica: el diseño del piloto y la expansión generaron la necesidad de realizar una revisión del modelo estático, para lo cual se realizó una reinterpretación del modelo estructural identificando nuevas fallas, y se realizó una correlación con alto nivel de detalle, logrando una mayor definición de las arenas (véase figura 13); lo anterior se consolidó a través de la implementación de una consultoría especializada en inyección de agua.

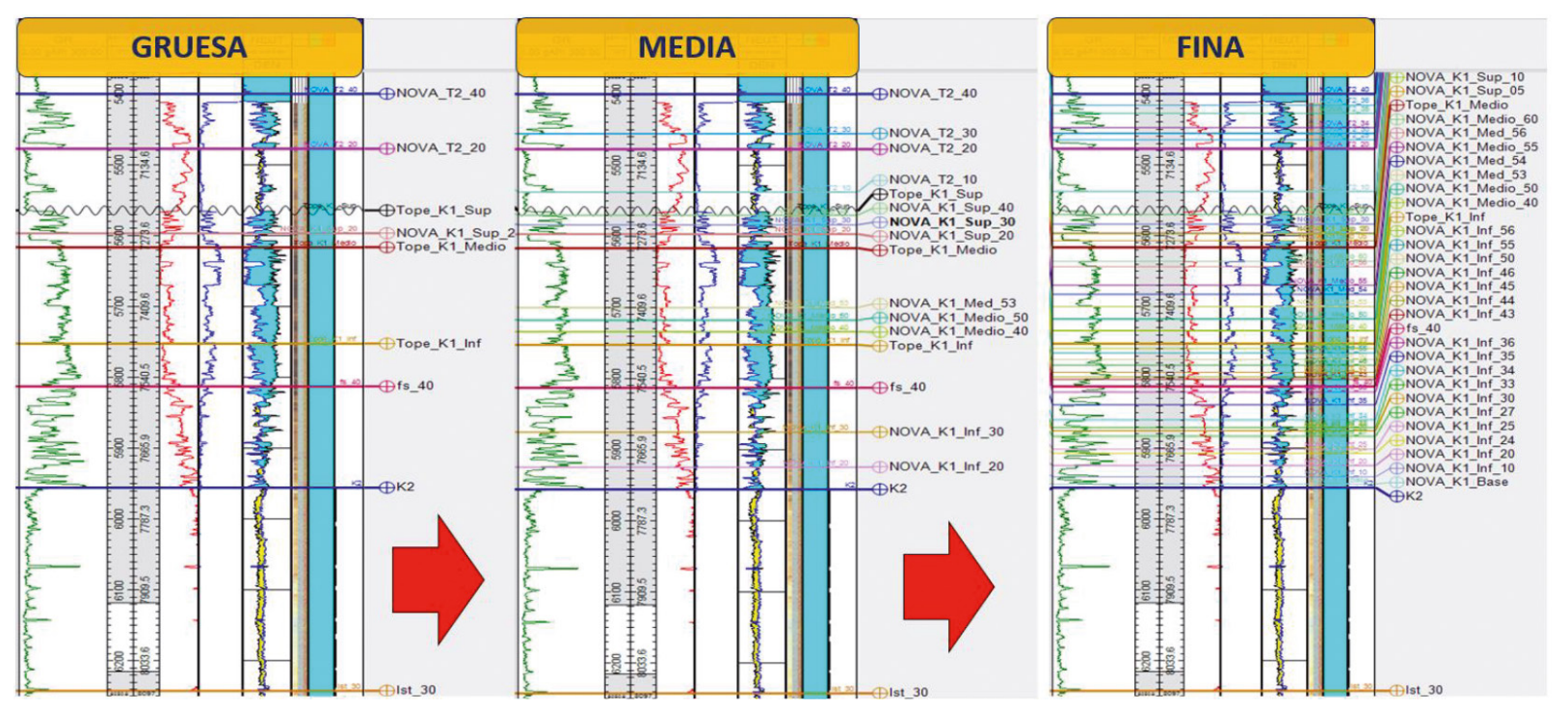

Figura 13. Ejemplo correlación de detalle formación K1. 
- Eficiencia vertical de la inyección: la primera etapa de inyección del pozo CAN05 se llevó a cabo sin sarta selectiva, lo cual ocasionó que el agua inyectada se distribuyera en las arenas de mejor calidad petrofísica, evidenciando la importancia de completar los pozos con sartas selectivas con el objetivo de mejorar la distribución vertical del barrido.

- Canalización temprana en pozos cercanos: los patrones de inyección en el campo corresponden a una geometría irregular, con pozos de distintos diseños (verticales, dirigidos y horizontales) y ubicados a una distancia entre $40 \mathrm{~m}$ y $500 \mathrm{~m}$ del inyector; adicional a esto, la relación de movilidad en el campo es desfavorable y se cuenta con una heterogenidad alta en la permeabilidad vertical de la formación K1 Inferior, lo que incrementa el riesgo de canalización en algunos pozos del patrón. El mecanismo de control para esta condición se logró mediante la regulación de válvulas en el pozo inyector y mediante el manejo de tasas de inyección y balance de las presiones fluyentes del patrón; eventualmente, la aplicación de conformance (químico o mecánico) en capas específicas. En la figura 14 se observa el comportamiento de un pozo canalizado, que logra recuperar su tendencia ajustando las presiones de fondo fluyente de los pozos productores pertenecientes al patrón.

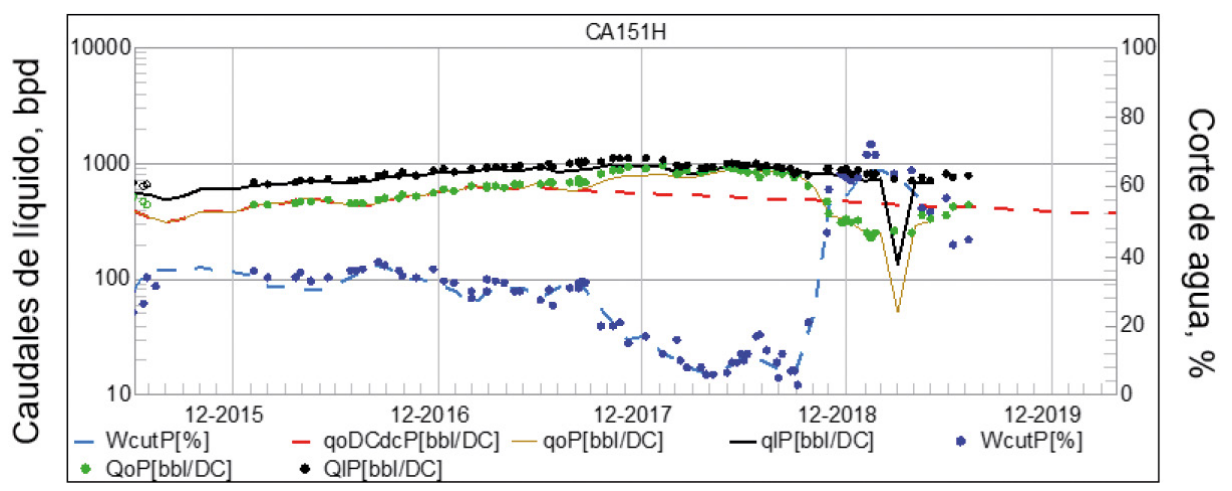

Figura 14. Ejemplo mitigación por canalización en pozos productores.

- Monitoreo calidad de agua: uno de los factores clave para lograr una eficiencia alta de inyección y un mantenimiento del índice de inyectividad de los pozos ha sido el monitoreo constante de la calidad del agua. Entre los parámetros que se miden se encuentran: concentración de grasas y aceites, concentración de sólidos suspendidos, tamaño de sólidos suspendidos, concentración de oxígeno, concentración de hierro, concentración de bacterias sulfato reductoras y productoras de ácido, concentración de $\mathrm{CO}_{2} \mathrm{y}_{2} \mathrm{~S}$, concentración de cloruros, turbidez, entre otros.

- Completamiento dual: dado que la formación K2 tiene un índice de productividad mucho mayor que la formación K1 Inferior, los pozos completados (commingled) no permiten un flujo apropiado de la formación K1 Inferior, incluso con el soporte de la inyección. Por tal razón, se probó el uso de completamientos duales con éxito, permitiendo producir las dos unidades independientemente.
- Superficie: el inicio de inyección en cada pozo se realizó mediante el alquiler de unidades temporales, que permitieron una mayor flexibilidad operacional (caudal/presión) y permitieron reducir la incertidumbre asociada al caudal de inyección para garantizar la eficiencia del proceso en cada patrón; posterior a esto, se efectúa la migración a instalaciones fijas de inyección en cada uno de los clústeres afectados.

\section{Conclusiones}

- Con la implementación del piloto de inyección de agua en el campo Castilla, en 13 patrones de inyección distribuidos a lo largo del campo, se logra la producción de 4,1 MMbls de aceite acumulados desde septiembre de 2015 a julio de 2019.

- La relación desfavorable de viscosidades entre el petróleo y el agua no ha sido impedimento para la implementación de esta alternativa de 
recobro, lo cual ha podido controlarse mediante bajas velocidades de inyección de agua; se evalúa una posible incorporación futura de polímeros para mejorar la eficiencia microscópica de desplazamiento.

- Aun cuando la ubicación y geometría de los pozos heredados del desarrollo primario no permitieron un patronamiento regular, el manejo de las tasas de inyección y el adecuado balance de presiones de fondo han permitido una respuesta positiva en la eficiencia areal de barrido.

- Debido a la alta heterogeneidad del yacimiento, fue necesaria la instalación de sartas selectivas para lograr una distribución adecuada del frente de barrido y mitigar el riesgo de canalizaciones tempranas, aumentando la recuperación de petróleo.

- El debido monitoreo de las variables que intervienen en un proceso de inyección de agua es indispensable para garantizar el éxito en el proceso en un yacimiento de crudo pesado.

\section{Reconocimientos}

El equipo técnico de desarrollo agradece la valiosa participación de los equipos de la gerencia técnica de yacimientos, proyectos, producción, completamiento, subsuelo, departamento de aseguramiento y control de la gerencia de recobro. Y, de manera especial, a la ingeniera Alexandra Velandia y al ingeniero Juan Sebastián Salazar por su importante contribución para este proyecto.

\section{Nomenclatura}

$\begin{array}{ll}\text { uo: } & \text { viscosidad del petróleo } \\ \text { uw: } & \text { viscosidad del agua } \\ \text { bwipd: } & \text { barriles de agua inyectados por día } \\ \text { EOR: } & \text { recuperación mejorada de petróleo } \\ \text { mD: } & \text { milidarcy } \\ \text { MM: } & \text { millones } \\ \text { OOIP: } & \text { petróleo original in situ } \\ \text { Pwf: } & \text { presión de fondo fluyente } \\ \text { TVD: } & \text { profundidad vertical verdadera } \\ \text { WAG: } & \text { agua alternando gas. }\end{array}$

\section{Referencias}

Beliveau, D. Waterflooding Viscous Oil Reservoirs. (2008). SPE-113132-MS.

Beliveau, D. Waterflooding Viscous Oil Reservoirs. (2009). SPE-113132-PA, Octubre 2009.

Compatibilidades entre Agua de Producción de la Estación Castilla y las de las Formaciones K1, K2 y T2 de Castilla (Factibilidad de Inyección de Agua de Producción). (2009). ICP, Ecopetrol.

Delamaide, E. Waterflooding Heavy Oil Reservoirs with Horizontal Wells: Field Examples from Canada. (2018). SPE-192087-MS.

Delamaide, E., et al. Pelican Lake Field: First Successful Application of Polymer Flooding In a Heavy-Oil Reservoir. (2014). SPE-165234-PA.

Depositional settings of the $\mathrm{K} 2, \mathrm{~K} 1$ and $\mathrm{T} 2$ reservoir systems in Castilla Field. (2010). ARC Group.

Depositional settings of the K1Sup reservoir systems in Castilla Field. (2015). ARC Group.

Evaluación Experimental de la Inyección de Agua en Campo Castilla Formaciones K2, K1 y T2. ICP. (2012). Ecopetrol.

Jackson, R. Why Waterfloods Fail. (1968). World Oil.

Kantzas, M., Kantzas, A., et al. Heavy Oil Waterflooding: Effectsof Flow Rate and Oil Viscosity. (2007). 8th Canadian International Petroleum Conference. JCPT.

Kantzas, M., Kantzas, A. Mechanism of Heavy Oil Recovery by Low Rate. (2008). Technology Symposium 2008 Joint Conference, Alberta, Canadá.

Kantzas, M., Kantzas, A. Improved Heavy Oil Recovery by Low Rate Waterflooding. (2008). SPE International Thermal Operations and Heavy Oil, Alberta, Canadá.

Mantilla, J., Rojas, D. Guía Maestra de Inyección de Agua. (2019). GOD-G-024, VDE, Ecopetrol.

Plan de Desarrollo Castilla. (2018) Ecopetrol.

Fecha de recepción: 28 enero de 2020

Fecha de aceptación: 30 de junio de 2021 